\title{
A Sarcopenia-Based Prediction Model for Postoperative Complications of ex vivo Liver Resection and Autotransplantation to Treat End-Stage Hepatic Alveolar Echinococcosis
}

\author{
Ting Sun' \\ Tao Wang' \\ Yiwen Qiu (iD) \\ Shu Shen' \\ Xianwei Yang' \\ Yi Yang' \\ Bin Huang ${ }^{2}$ \\ Wentao Wang (D) \\ 'Department of Liver Surgery \& Liver \\ Transplantation Center, West China \\ Hospital of Sichuan University, Chengdu, \\ People's Republic of China; ${ }^{2}$ Department \\ of Vascular Surgery, West China Hospital \\ of Sichuan University, Chengdu, People's \\ Republic of China
}

Correspondence: Wentao Wang

Email wwtdoctor02@163.com
Background: Sarcopenia and visceral adiposity have been shown to be associated with postoperative complications in numerous diseases. However, their effects on the postoperative complications of end-stage hepatic alveolar echinococcosis (HAE) patients undergoing ex vivo liver resection and autotransplantation (ELRA) remain unclear.

Methods: This retrospective study included 101 end-stage HAE patients who underwent ELRA from January 2014 to August 2020. We measured the skeletal muscle and adipose tissue of all patients at the level of the third lumbar vertebra on plain abdominal computed tomography (CT) images and subsequently derived an equation via least absolute shrinkage and selection operator (LASSO) regression analysis to calculate the sarcopenia score. Univariate and multivariate regression were performed to reveal the relationship between major postoperative complications and perioperative clinical data, and the obtained nomogram was validated with the bootstrapping method.

Results: The sarcopenia score was constructed as a personalized indicator to evaluate sarcopenia and visceral adiposity in each patient. Logistic regression analysis finally selected duration from primary diagnosis to obvious symptoms (OR=1.024, 95\% CI, 1.007-1.042), surgical time $(\mathrm{OR}=1.003,95 \% \mathrm{CI}, 0.999-1.007)$ and sarcopenia score $(\mathrm{OR}=4.283,95 \% \mathrm{CI}$, $1.739-10.551)$ as independent risk factors for predicting major postoperative complications following ELRA for end-stage HAE patients. The area under the receiver operating characteristic curve (AUROC) of 0.807 (95\% CI, 0.720-0.895) and the calibration curve for this prediction model were satisfactory.

Conclusion: The sarcopenia score, which systematically evaluates the skeletal muscle and adipose tissue of end-stage HAE patients, was a significant predictive factor for major postoperative complications of ELRA. Relevant interventions should be conducted for those who have a high risk of postoperative complications according to the nomogram.

Keywords: hepatic alveolar echinococcosis, liver transplantation, hepatectomy, sarcopenia, postoperative complications

\section{Introduction}

Hepatic alveolar echinococcosis (HAE), a zoonotic disease caused by Echinococcus multilocularis (Em) infection with approximately 18,235 new cases per year in China, Central Asia, Europe and some other areas, is potentially lethal to humans and is also called a "parasitic cancer". ${ }^{1-4}$ HAE, especially end-stage HAE, is frequently characterized by infiltrative growth that results in invasion into crucial 
intrahepatic structures, such as vascular invasion and bile duct invasion. In general, surgical resection and oral albendazole therapy are recommended for HAE. ${ }^{5}$ However, as a result of the occult onset and infiltrative growth of this condition, most patients usually lose the opportunity to undergo radical surgery and need to receive complex liver surgery or palliative treatment.

Ex vivo liver resection and autotransplantation (ELRA), a complicated surgery that includes complete hepatectomy, ex vivo liver resection and autologous transplantation, has been considered an effective treatment for end-stage HAE in recent years. ${ }^{6-8}$ In 1988, Pilchmayr et $\mathrm{al}^{9}$ first reported extracorporeal liver surgery and its application for advanced malignant tumors. However, the outcome of the surgical technique was unsatisfactory. ${ }^{10}$ In contrast, end-stage HAE, as a benign disease, has become a potential indicator for ELRA. ${ }^{11}$ ELRA involves the precise resection of lesions, meticulous repair of hepatic vessels and complex reconstruction of conduits, together with complicated maintenance anesthesia and ex vivo organ perfusion, making the surgical procedure stressful and challenging. The difficulty of ELRA for end-stage HAE raises great concern regarding the occurrence of postoperative complications, which greatly impact the quality of life and overall survival of patients.

In modern surgical operations, surgeons and researchers pay increasing attention to the role of nutritional status in the preoperative assessment, and malnutrition has been proven to be a risk factor for postoperative complications. ${ }^{12,13}$ Sarcopenia, a malnutrition-related muscle disease characterized by low muscle strength, low muscle quantity and quality and poor physical performance, mainly occurs in older people and is possibly caused by disordered energy metabolism. ${ }^{14}$ Several studies have reported that sarcopenia, myosteatosis, visceral adiposity and other malnutrition-related indicators play vital roles in the development of postoperative complications. $^{15-28}$ The Clavien-Dindo classification ${ }^{29}$ of surgical complications is widely recognized and current studies have found that common postoperative complications of liver surgery include biliary leakage, surgical site infections, ascites, etc. $^{30-32}$ However, there is no research on the impact of sarcopenia on ELRA complications or on prediction models for postoperative complications of ELRA.

As our hospital is the nearest medical center from one of the main HAE epidemic areas in the world, we conducted a series of analyses based on our ELRA cases for end-stage HAE to fill these gaps. In this study, we analyzed a large amount of patient information, explored possible risk factors for postoperative complications of ELRA, revealed the relationship between sarcopenia combined with adipose tissue and postoperative complications, and developed a nomogram to offer a convenient and quantitative approach to predict and estimate the risk of complications after ELRA for end-stage HAE patients.

\section{Methods}

\section{Patients}

This retrospective study consecutively enrolled a total of 101 end-stage HAE patients (age $\geq 18$ years) who underwent ELRA at West China Hospital of Sichuan University from January 2014 to August 2020. The diagnostic standard of $\mathrm{HAE}$ and its PNM staging system comply with the guidelines of the World Health Organization (WHO). ${ }^{33}$ It is worth mentioning that end-stage HAE was defined as HAE with vascular invasion, including into the inferior vena cava (IVC), portal vein (PV) and hepatic arteries, with or without adjacent organ invasion and distant metastasis (P4, N0 or N1, M0 or M1). All 101 patients were strictly evaluated by a multidisciplinary team including hepatobiliary surgeons, a vascular surgeon, a radiologist, and an anesthetist, and the following key features of all patients undergoing ELRA were noted: (1) involvement of the hepatic hilar region, IVC, and 3 hepatic veins (HVs) or invasion into the secondary or tertiary branches of the PVs and hepatic arteries requiring complicated reconstruction with an extended ischemic time that the liver might not tolerate; (2) good physiological status and relatively normal liver and kidney function (total bilirubin level lower than twice the upper limit of normal) with or without extrahepatic echinococcosis lesions that could be controlled by albendazole or be completely removed by surgery; and (3) an estimated remnant liver volume (RLV)/ standard liver volume (SLV) ratio greater than 0.4.

The study was conducted in accordance with the Declaration of Helsinki and was approved by the Ethics Committee of West China Hospital of Sichuan University (No. 2017-38). The requirement for informed consent was waived because the study was retrospective, and the data were anonymized.

\section{Preoperative Evaluation and Image Analysis}

We evaluated the characteristics of the HAE lesions, relevant incidences of vascular invasion and extrahepatic metastasis by computed tomography (CT) and magnetic resonance imaging (MRI). The RLV was calculated by a three-dimensional 
(3D) imaging analysis system ${ }^{34}$ (CAS; Qingdao Hisense Medical Equipment Co., Ltd., Qingdao, P. R. China), and the SLV was calculated according to the method described by Tongyoo et $\mathrm{al}^{35}$ and Urata et al. ${ }^{36}$ The classification of vascular infiltration (invasion into the IVC, HV and PV) followed the definitions in a previous study by Qiu et al. ${ }^{6}$

We specifically collected plain abdominal CT images of all patients within one month before ELRA. Then, analyses of the image data and measurements of skeletal muscle and adipose tissue were performed at the level of the third lumbar vertebra with three-dimensional visualization software ${ }^{28}$ (Mimics Interactive Medical Image Control System, Version 25.0, Materialize Company, Belgium). To divide out skeletal muscle and adipose tissue at the level of the third lumbar vertebra, skeletal muscle areas, which included the psoas, erector spinae, quadratus lumborum, transversus abdominis, and internal and external obliques, were quantified using -29 to 150 Hounsfield units (HU), and adipose tissue areas, which included the subcutaneous adipose tissue and visceral adipose tissue, were quantified using -190 to -30 HU. ${ }^{37}$ The sarcopenia status of all patients could be measured and calculated by the following parameters: psoas muscle mass index (PMI), skeletal muscle mass index (SMI), visceral to subcutaneous adipose tissue area ratio (VSR), and intramuscular adipose tissue content (IMAC). The calculations were as follows: $\mathrm{PMI}=$ left and right psoas muscle area $\left(\mathrm{cm}^{2}\right)$ patient's height ${ }^{2}\left(\mathrm{~m}^{2}\right)$; SMI $=$ skeletal muscle area $\left(\mathrm{cm}^{2}\right) /$ patient's height ${ }^{2}\left(\mathrm{~m}^{2}\right)$; and $\mathrm{VSR}=$ visceral adipose tissue area $\left(\mathrm{cm}^{2}\right) /$ subcutaneous adipose tissue area $\left(\mathrm{cm}^{2}\right)$. IMAC was calculated as the CT value of the multifidus muscles (HU)/CT value of the subcutaneous fat (HU). The average CT value of two regions of interest (ROIs) placed on the multifidus muscles at the L3 level was defined as the $\mathrm{CT}$ value of the multifidus muscles. The average CT value of four ROIs placed on the subcutaneous fat at the L3 level was defined as the CT value of subcutaneous fat. ${ }^{28}$

The patients' demographic data, basic lesion information, medical history data, surrounding invasions, distant metastases, vascular infiltrations, liver function, and estimated RLV and SLV were collected.

\section{ELRA Procedures}

The surgical procedures mainly consisted of three parts. The first part included exploratory surgery and combined resection of the entire liver and invaded surrounding organs or tissues. In the second part, ex vivo liver resection of the HAE lesions and repair of the major conduits were performed with $0-4{ }^{\circ} \mathrm{C}$ organ perfusion. At the same time, we temporarily reconstructed the IVC and established a portocaval shunt. Finally, the autograft was reimplanted, and the conduits were reconstructed. The detailed steps of ELRA have been reported by Yang et al. ${ }^{7}$

During surgery, data on the combined resection, blood transfusion volume, time that each step of the surgery took and actual RLV were recorded. The hemodynamic instability time was defined as the time of IVC and PV occlusion.

\section{Postoperative Follow-Up}

Follow-up abdominal $\mathrm{CT}$ and other routine inspections were performed every 3-6 months after hospital discharge to detect potential complications and recurrence. Postoperative morbidity was measured by Clavien-Dindo grade $^{38}$ and the comprehensive complication index (CCI). ${ }^{39}$

The length of stay in the hospital and in the intensive care unit (ICU) were recorded.

\section{Statistical Analysis}

Parametric continuous data are presented as the mean \pm standard deviation (SD), and nonparametric continuous data are presented as the median and interquartile ranges. Categorical data are presented as numbers and percentages. Least absolute shrinkage and selection operator (LASSO) logistic regression was used to explore and show the correlation between postoperative complications and sarcopenia. Logistic regression analysis was utilized for multivariate analysis [odds ratio (OR), 95\% confidence interval (CI)]. A nomogram to predict major postoperative complications containing sarcopenia score and other risk factors was constructed using stepwise selection and the Akaike information criterion (AIC). The stepwise selection method was used to select variables and determine model fit. AIC was used for model evaluation and model selection. A receiver operating characteristic (ROC) curve and area under the receiver operating characteristic curve (AUROC) were utilized to analyze the predictive performance of the model. The calibration curve was derived from regression analysis, and the number of bootstrap resamples was 500. Median values in each tertile and quartile were used to test for a linear trend. All the reported $p$ values are 2-tailed, and $p<0.05$ was considered statistically significant. All statistical analyses were performed using Empower ${ }^{\circledR}$ (www.empowerstats.com, X\&Y Solutions Inc., Boston MA). 


\section{Results}

\section{Patient Characteristics}

A total of 101 end-stage HAE patients, including 38 male and 63 female patients, underwent ELRA after receiving a comprehensive evaluation; their mean age was 37 years. All the required preoperative, intraoperative and postoperative follow-up data were collected and analyzed. The clinical data and perioperative characteristics are shown in Table 1. In this study, the mean preoperative PMI, SMI, VSR and IMAC were $5.56 \mathrm{~cm}^{2} / \mathrm{m}^{2}, 41.35 \mathrm{~cm}^{2} / \mathrm{m}^{2}, 0.28$ and -0.57 , respectively. The median CCI of all patients was 20.92 . Postoperative complications developed in 57 patients (56.44\%; grade $\mathrm{I}=3$, grade $\mathrm{II}=15$, grade $\mathrm{III}=28$, grade $\mathrm{IV}=0$, grade $\mathrm{V}=11$ ), and major postoperative complications (Clavien-Dindo grade $\geq$ IIIa) occurred in 39 patients $(38.61 \%)$. Among all patients, the three most common postoperative complications were biliary leakage $(n=21)$, pleural effusion $(n=9)$ and ascites $(n=9)$.

\section{LASSO Logistic Regression for the Relationship Between Sarcopenia and Postoperative Complications}

The LASSO logistic regression analysis initially included sex, age, weight, PMI, SMI, VSR and IMAC and finally selected four significant variables: SMI, VSR, IMAC and age. Ten-fold cross-validation was used to select the tuning parameters, and the minimum mean cross-validated error was calculated. Based on the coefficients obtained from the LASSO algorithm when value of $\lambda$ gives minimum mean crossvalidation error $[\log (\lambda)=-3.317]$, we finally constructed the formula as follows: sarcopenia score $=$ $0.287-0.024 \times \mathrm{SMI}+1.761 \times \mathrm{VSR}+1.703 \times \mathrm{IMAC}$ $+0.018 \times$ age (sarcopenia score calculator shown later). The AUROC, mean cross-validation error and standard deviation of mean cross-validation error were $0.723,1.283$ and 0.061 for $\log (\lambda)=-3.317$. The LASSO coefficient profiles of these variables and their related partial likelihood deviance are plotted in Figure 1. The sarcopenia score indicated a regression relationship between major postoperative complications and the physical condition of skeletal muscle and adipose tissue. A higher sarcopenia score denoted a lower quantity and quality of skeletal muscle and a larger amount of adipose tissue.

\section{Logistic Regression Analyses and Construction of a Prognostic Nomogram to Predict Postoperative Complications of ELRA}

To identify the risk factors for major postoperative complications, we performed univariate regression analyses (Table 2). The analysis showed that the duration from primary diagnosis to obvious symptoms, distant metastasis, surgical time, anhepatic time and sarcopenia score were associated with the odds of major postoperative complications of ELRA. Then, these five variables were included in a multivariate regression analysis (Table 2), from which duration from primary diagnosis to obvious symptoms $(\mathrm{OR}=1.024,95 \% \mathrm{CI}, 1.007-1.042)$, surgical time $(\mathrm{OR}=1.003,95 \% \mathrm{CI}, 0.999-1.007)$ and sarcopenia score $(\mathrm{OR}=4.283,95 \% \mathrm{CI}, 1.739-10.551)$ were selected as the variables for the final logistic regression model based on stepwise selection and AIC values.

Based on LASSO logistic regression and the multivariate regression analysis, a prognostic nomogram for predicting the occurrence of major postoperative complications in ELRA patients was built (Figure 2). Internal validation was performed via the bootstrapping method (resample: 500), and the relevant ROC curve and calibration curve were generated (Figure 3). The ROC curve is displayed in Figure 3A, with a relatively satisfactory AUROC of 0.807 (95\% CI, 0.720-0.895). In addition, the calibration curve in Figure 3B shows close agreement between the predictions of the nomogram and actual major postoperative complications. The calculator of sarcopenia score and predicted risk of major postoperative complications is available in the Supplementary file 1.

To assess nonlinearity, we experimented with different transformations of duration from primary diagnosis to obvious symptoms, surgical time and sarcopenia score (specifically, using a logarithm or a quadratic term). Despite these transformations, the results did not change (data not shown). The linearities of the three variables were tested by a triple-term ( $\mathrm{p}$ for trend $=0.006$ for duration from primary diagnosis to obvious symptoms; $p$ for trend $=0.033$ for surgical time; $p$ for trend $=0.008$ for sarcopenia score) and quartic-term ( $\mathrm{p}$ for trend $=0.008$ for duration from primary diagnosis to obvious symptoms; $p$ for trend $=0.044$ for surgical time; $p$ for trend $=0.002$ for sarcopenia score) transformation.

According to the ROC curves, we classified patients into the low-risk group and the high-risk group with 
Table I Clinical Data and Surgical Characteristics of the I0I Patients

\begin{tabular}{|c|c|}
\hline Variable & Value \\
\hline \multicolumn{2}{|c|}{ Baseline data } \\
\hline Number of patients, $n$ & 101 \\
\hline Age (years), mean \pm SD & $37.00 \pm 9.97$ \\
\hline $\mathrm{BMI}\left(\mathrm{kg} / \mathrm{m}^{2}\right)$, mean $\pm \mathrm{SD}$ & $22.05 \pm 3.21$ \\
\hline Duration from primary diagnosis to obvious symptoms (months), mean $\pm S D$ & $30.80 \pm 33.05$ \\
\hline Male sex, n (\%) & $38(37.62 \%)$ \\
\hline Previous use of oral albendazole, $n(\%)$ & $65(64.36 \%)$ \\
\hline History of jaundice, $n(\%)$ & $18(17.82 \%)$ \\
\hline \multicolumn{2}{|c|}{ Preoperative data of skeletal muscle and adipose tissue } \\
\hline $\mathrm{PMI}\left(\mathrm{cm}^{2} / \mathrm{m}^{2}\right)$, mean $\pm \mathrm{SD}$ & $5.56 \pm 2.07$ \\
\hline $\mathrm{SMI}\left(\mathrm{cm}^{2} / \mathrm{m}^{2}\right)$, mean $\pm \mathrm{SD}$ & $41.35 \pm 8.47$ \\
\hline $\operatorname{VSR}\left(\mathrm{cm}^{2} / \mathrm{cm}^{2}\right)$, mean $\pm \mathrm{SD}$ & $0.28 \pm 0.18$ \\
\hline IMAC $(H U / H U)$, mean \pm SD & $-0.57 \pm 0.18$ \\
\hline \multicolumn{2}{|c|}{ Preoperative data of the lesions } \\
\hline Lesion diameter $(\mathrm{cm})$, mean $\pm \mathrm{SD}$ & $15.17 \pm 2.72$ \\
\hline Surrounding invasion, $\mathrm{n}(\%)$ & $68(67.33 \%)$ \\
\hline Distant metastasis, n (\%) & $16(15.84 \%)$ \\
\hline \multicolumn{2}{|l|}{ Number of lesions, $n(\%)$} \\
\hline Single & $59(58.42 \%)$ \\
\hline Multiple & $42(4 I .58 \%)$ \\
\hline \multicolumn{2}{|l|}{ Inferior vena cava invasion, $\mathrm{n}(\%)$} \\
\hline I & $8(7.9208 \%)$ \\
\hline II & 77 (76.24\%) \\
\hline III & $16(15.84 \%)$ \\
\hline \multicolumn{2}{|l|}{ Hepatic vein invasion, $n(\%)$} \\
\hline 1 & $43(42.57 \%)$ \\
\hline II & $58(57.43 \%)$ \\
\hline \multicolumn{2}{|l|}{ Portal vein invasion, $\mathrm{n}(\%)$} \\
\hline I & $7(6.93 \%)$ \\
\hline II & $68(67.33 \%)$ \\
\hline III & $26(25.74 \%)$ \\
\hline \multicolumn{2}{|c|}{ Preoperative data of liver volume and quality } \\
\hline $\operatorname{ERLV}(\mathrm{mL})$, mean $\pm \mathrm{SD}$ & $712.85 \pm 198.65$ \\
\hline $\mathrm{SLV}(\mathrm{mL})$, mean $\pm \mathrm{SD}$ & $1147.62 \pm 109.17$ \\
\hline ERLV/SLV, mean $\pm S D$ & $0.62 \pm 0.17$ \\
\hline \multicolumn{2}{|l|}{ Child-Pugh classification, $\mathrm{n}(\%)$} \\
\hline Class A & 95 (94.06\%) \\
\hline Class B & $6(5.94 \%)$ \\
\hline \multicolumn{2}{|l|}{ Albumin-bilirubin grade, $\mathrm{n}(\%)$} \\
\hline Grade I $(\leq-2.60)$ & $23(22.77 \%)$ \\
\hline Grade $2(>-2.60, \leq-1.39)$ & $76(75.25 \%)$ \\
\hline Grade 3 (>-1.39) & $2(1.98 \%)$ \\
\hline
\end{tabular}

(Continued) 
Table I (Continued).

\begin{tabular}{|c|c|}
\hline Variable & Value \\
\hline \multicolumn{2}{|c|}{ Intraoperative data } \\
\hline $\operatorname{ARLV}(m L)$, mean $\pm S D$ & $723.01 \pm 207.78$ \\
\hline ARLV/SLV, mean \pm SD & $0.63 \pm 0.18$ \\
\hline Surgical time $(\mathrm{min})$, mean $\pm S D$ & $744.74 \pm \mid 37.11$ \\
\hline Anhepatic time (30 min), mean $\pm S D$ & $10.34 \pm 2.62$ \\
\hline Hemodynamic instability time $(\min )$, mean $\pm S D$ & $148.87 \pm 35.30$ \\
\hline Liver graft reimplantation time ( $10 \mathrm{~min})$, mean \pm SD & $5.96 \pm 2.42$ \\
\hline Blood loss $(\mathrm{mL})$, mean \pm SD & $2608.91 \pm 1273.88$ \\
\hline Autologous blood transfusion ( $100 \mathrm{~mL})$, mean $\pm \mathrm{SD}$ & $9.35 \pm 9.78$ \\
\hline RBC suspension transfusion $(U)$, mean $\pm S D$ & $7.76 \pm 6.85$ \\
\hline Fresh frozen plasma transfusion $(\mathrm{mL})$, mean $\pm \mathrm{SD}$ & $930.99 \pm 1013.55$ \\
\hline Combined resection, $\mathrm{n}(\%)$ & $22(21.78 \%)$ \\
\hline \multicolumn{2}{|c|}{ Length of stay and postoperative complications } \\
\hline Length of stay in the hospital (days), median (range) & $20.00(15.00-31.00)$ \\
\hline Length of stay in the ICU (days), median (range) & $4.00(3.00-5.00)$ \\
\hline Postoperative complication, $\mathrm{n}(\%)$ & 57 (56.44\%) \\
\hline Postoperative complication (Clavien-Dindo $\geq$ IIla), n (\%) & $39(38.61 \%)$ \\
\hline $\mathrm{CCl}$, median (range) & $20.92(0-26.22)$ \\
\hline
\end{tabular}

Abbreviations: BMI, body mass index; PMI, psoas muscle mass index; SMI, skeletal muscle mass index; VSR, visceral to subcutaneous adipose tissue area ratio; IMAC, intramuscular adipose tissue content; ERLV, estimated remnant liver volume; SLV, standard liver volume; ARLV, actual remnant liver volume; RBC, red blood cell; ICU, intensive care unit; $\mathrm{CCl}$, comprehensive complication index.

a predicted probability of 0.3298 as the optimal cut-off value. 52 patients $(51.49 \%)$ were classified into the lowrisk group and 49 patients (48.51\%) were classified into the high-risk group (Table 3). As expected, duration from primary diagnosis to obvious symptoms ( 15.65 vs 46.88 months, $\mathrm{p}<0.001)$, surgical time (677.52 vs $816.08 \mathrm{~min}$, $\mathrm{p}<0.001)$ and sarcopenia score $(-0.88$ vs $-0.09, \mathrm{p}<0.001)$ showed significant differences between the two groups. In addition, significant between-group differences were observed in age, PMI, SMI, VSR, IMAC, surrounding invasion, portal vein invasion, anhepatic time, hemodynamic instability time, fresh frozen plasma transfusion, length of stay in the ICU, CCI and postoperative complication. No other between-group differences were found.

\section{Discussion}

HAE, as a lethal infectious disease caused by a parasite, necessitates further attention, especially in developing countries with a high prevalence of alveolar echinococcosis (AE). ${ }^{40}$ Due to tumor-like characteristics such as hidden onset and vascular invasions, the mortality rate of HAE has been reported to be as high as $90 \%$ if untreated for 10 to 15 years. ${ }^{11}$ Although radical resection surgery combined with albendazole is recognized as the best therapy strategy, especially for advanced HAE, ${ }^{41}$ approximately a quarter of HAE patients still lose the opportunity to undergo normal radical surgery due to the presence of end-stage lesions. ${ }^{11}$ For such patients who were diagnosed and treated late, palliative resection, oral albendazole and liver transplantation were the only three possible treatment methods. However, palliative treatment for end-stage HAE showed little success according to Kern, ${ }^{42}$ and the research from $\mathrm{Qu}$ et $\mathrm{al}^{43}$ showed that the five-year survival rate with long-term albendazole was only $10 \%-40 \%$. On the other hand, liver allotransplantation provided a chance for survival for patients with "unresectable" HAE, ${ }^{44-46}$ but the results seemed to be underwhelming. Bressom-Handi et $\mathrm{al}^{47}$ summarized 47 cases of liver transplantation for HAE, and the five-year survival rate without recurrence was $58 \%$. In a single-center experience including 10 living donor liver transplantation (LDLT) cases, Ozdemir et $\mathrm{al}^{44}$ showed that the local recurrence and distant metastasis rates were $10 \%$ and $20 \%$, respectively. Furthermore, the shortage of donor organs, high surgical costs and lifetime of immunosuppressive treatment remain stumbling blocks for allotransplantation therapy for end-stage HAE. Because of these factors, the possibility of treating endstage HAE with ELRA arose. 


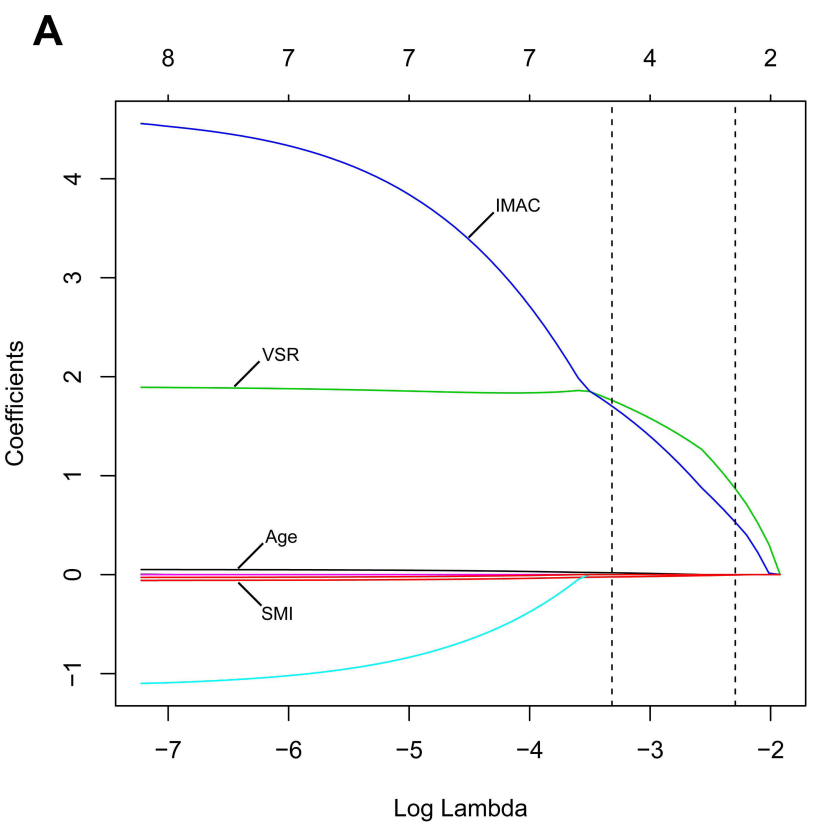

B

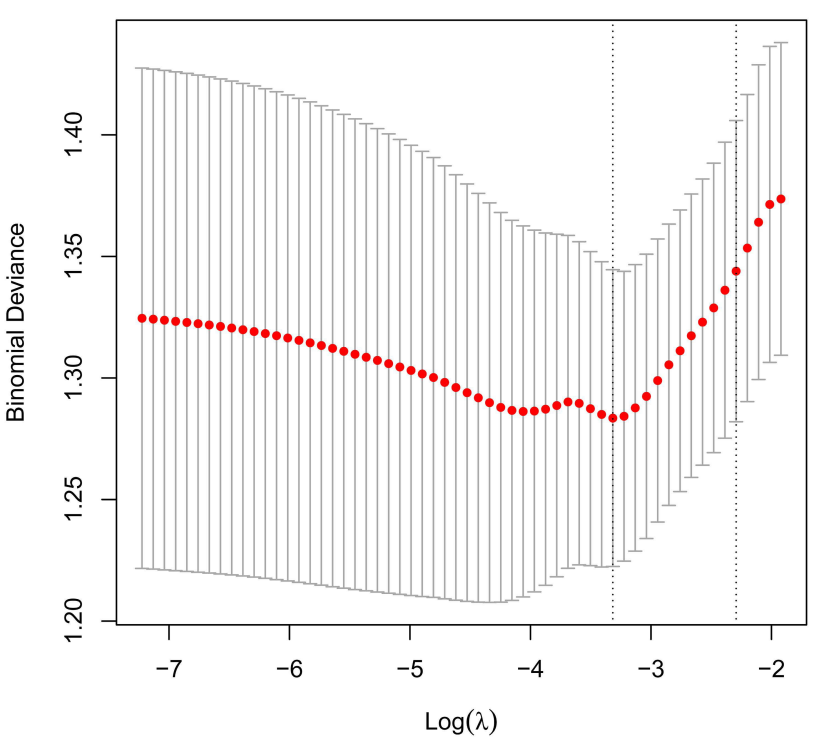

Figure I (A) Least absolute shrinkage and selection operator (LASSO) coefficient profiles of four sarcopenia-associated variables. IMAC, VSR, age and SMI were finally selected as significant variables by LASSO logistic regression. The blue line represents IMAC, the green line represents VSR, the black solid line represents age, and the bottom red line represents SMI. A dashed vertical line is drawn at $\log (\lambda)=-3.317$, which was chosen by I0-fold cross-validation. IMAC, intramuscular adipose tissue content; VSR, visceral to subcutaneous adipose tissue area ratio; SMI, skeletal muscle mass index. (B) The partial likelihood deviance (binomial deviance) for the LASSO coefficient profiles. The red dots represent the average partial likelihood deviances for every model with a given lambda. A dotted vertical line represents the partial likelihood deviance error at $\log (\lambda)=-3.317$.

Since the surgical technique of ELRA was used to treat end-stage HAE in $2011,{ }^{48}$ an increasing number of ELRA procedures for HAE have been reported by medical centers. ${ }^{7,11,49-52}$ For ELRA, the limitations of liver allotransplantation become strengths, including no need for donors and immunosuppressive drugs as well as lower therapy costs. As ELRA involves a series of complex surgical procedures, postoperative complications become more of a concern. This study is the largest scale analysis on postoperative complications of ELRA for end-stage HAE. In our study, major postoperative complications occurred in $38.61 \%$ of all patients, which is higher than the $22 \%$ reported by Qiu et $\mathrm{al}^{6}$ and $14 \%$ by Aji et $\mathrm{al}^{11}$ and we managed most complications well. We attribute the higher rate of major postoperative complications partly to the expanded indications and partly to the relatively later disease stage. Postoperative complications always cause distress to the patients, increase therapy costs and even affect prognosis. ${ }^{53}$ It is therefore necessary to pay more attention to the possible risk factors for postoperative complications.

A consensus definition of sarcopenia was first formally presented by the European Working Group on Sarcopenia in Older People (EWGSOP) in 2010. ${ }^{54}$ Sarcopenia has been shown to be a risk factor for postoperative complications in many diseases, such as hepatocellular carcinoma (HCC), pancreatic cancer and other gastrointestinal cancers. ${ }^{55-58}$ In addition, many studies have revealed that sarcopenia plays a vital role in liver transplantation and is even associated with surgical morbidity. ${ }^{15-18}$ On the basis of the definition of sarcopenia, sarcopenia is closely associated with the amount of skeletal muscle, its function and its impacts on physical performance. As a result, PMI and SMI, which are easy to measure at the third lumbar vertebra and are not easily influenced by ascites fluid, have been widely utilized to evaluate the degree of sarcopenia. ${ }^{59-61}$ IMAC, representing the amount of adipose tissue in the multifidus muscles, can reflect the quality of skeletal muscle and was also calculated. ${ }^{62}$ Furthermore, VSR, which indicates visceral adiposity, was found to be an important factor for adverse outcomes in HCC by Fujiwara et al. ${ }^{19}$ Thus, we included PMI, SMI, IMAC and VSR coupled with baseline physical data, including age, sex and weight, to assess the sarcopenia score of each end-stage HAE patient. In addition, we adopted LASSO logistic regression to calculate the sarcopenia score, which was simultaneously assessed by sarcopenia-related indicators and visceral adiposity and thus avoided the inclusion of redundant parameters or overfitting of the model. It is noteworthy that the sarcopenia 
Table 2 Logistic Regression Analyses for Major Postoperative Complications

\begin{tabular}{|c|c|c|c|c|c|c|}
\hline \multirow[t]{2}{*}{ Variable } & \multicolumn{3}{|c|}{ Univariate Regression } & \multicolumn{3}{|c|}{ Multivariate Regression } \\
\hline & OR & $95 \% \mathrm{Cl}$ & $\mathbf{P}$ & OR & $95 \% \mathrm{Cl}$ & $\mathbf{P}$ \\
\hline \multicolumn{7}{|c|}{ Baseline data } \\
\hline Duration from primary diagnosis to obvious symptoms (months) & 1.026 & $1.010-1.042$ & 0.001 & 1.024 & $1.007-1.042$ & 0.006 \\
\hline Previous use of oral albendazole (yes vs, no) & 1.421 & $0.607-3.327$ & 0.418 & & & \\
\hline History of jaundice (yes vs no) & 1.767 & $0.633-4.933$ & 0.277 & & & \\
\hline \multicolumn{7}{|c|}{ Preoperative data of skeletal muscle and adipose tissue } \\
\hline Sarcopenia score & 4.872 & $2.060-11.525$ & 0.000 & 4.283 & I.739-10.55। & 0.002 \\
\hline \multicolumn{7}{|l|}{ Preoperative data of the lesions } \\
\hline Lesion diameter $(\mathrm{cm})$ & 1.009 & $0.870-1.169$ & 0.909 & & & \\
\hline Surrounding invasion (yes vs no) & 1.710 & $0.706-4.141$ & 0.234 & & & \\
\hline Distant metastasis (yes vs no) & 3.218 & I.064-9.737 & 0.039 & 2.326 & $0.57 I-9.484$ & 0.239 \\
\hline Number of lesions (multiple vs single) & 0.963 & $0.427-2.173$ & 0.928 & & & \\
\hline \multicolumn{7}{|l|}{ IVC invasion } \\
\hline II vs I & 1.007 & $0.224-4.530$ & 0.993 & & & \\
\hline III vs I & 1.296 & $0.228-7.379$ & 0.770 & & & \\
\hline HV invasion (II vs I) & 1.875 & $0.816-4.306$ & 0.138 & & & \\
\hline \multicolumn{7}{|l|}{ PV invasion } \\
\hline II vs I & 1.364 & $0.246-7.567$ & 0.723 & & & \\
\hline III vs I & 2.500 & $0.409-15.293$ & 0.321 & & & \\
\hline \multicolumn{7}{|c|}{ Preoperative data of liver volume and quality } \\
\hline ERLV (mL) & 1.001 & $0.999-1.003$ & 0.230 & & & \\
\hline SLV (mL) & 0.999 & $0.995-1.003$ & 0.636 & & & \\
\hline ERLV/SLV & 6.216 & $0.570-67.747$ & 0.134 & & & \\
\hline Child-Pugh classification (class B vs class $A$ ) & 0.784 & $0.137-4.496$ & 0.785 & & & \\
\hline \multicolumn{7}{|l|}{ ALBI grade } \\
\hline Grade 2 vs grade 1 & 2.763 & $0.929-8.215$ & 0.068 & & & \\
\hline Grade 3 vs grade I & 3.600 & $0.190-68.344$ & 0.394 & & & \\
\hline \multicolumn{7}{|c|}{ Intraoperative data } \\
\hline ARLV $(m L)$ & 1.001 & $0.999-1.003$ & 0.513 & & & \\
\hline ARLV/SLV & 2.869 & $0.287-28.686$ & 0.370 & & & \\
\hline Surgical time $(\mathrm{min})$ & 1.004 & $1.001-1.008$ & 0.009 & 1.003 & $0.999-1.007$ & 0.106 \\
\hline Anhepatic time (30 min) & 1.251 & $1.056-1.482$ & 0.009 & 1.057 & $0.817-1.369$ & 0.672 \\
\hline Hemodynamic instability time (min) & 0.993 & $0.98 \mathrm{I}-1.005$ & 0.222 & & & \\
\hline Liver graft reimplantation time ( $10 \mathrm{~min})$ & 0.851 & $0.711-1.019$ & 0.079 & & & \\
\hline Blood loss $(\mathrm{mL})$ & 1.000 & $1.000-1.001$ & 0.073 & & & \\
\hline Autologous blood transfusion $(\mathrm{mL})$ & 1.038 & $0.995-1.082$ & 0.087 & & & \\
\hline RBC suspension transfusion $(U)$ & 1.039 & $0.979-1.103$ & 0.206 & & & \\
\hline Fresh frozen plasma transfusion $(\mathrm{mL})$ & 1.000 & $1.000-1.000$ & 0.683 & & & \\
\hline Combined resection (yes vs no) & 2.311 & $0.885-6.032$ & 0.087 & & & \\
\hline
\end{tabular}

Abbreviations: OR, odds ratio; $\mathrm{Cl}$, confidence interval; IVC, inferior vena cava; $\mathrm{HV}$, hepatic vein; PV, portal vein; ERLV, estimated remnant liver volume; SLV, standard liver volume; ALBI, albumin-bilirubin; ARLV, actual remnant liver volume; RBC, red blood cell.

score was not merely an independent risk factor for major postoperative complications but could also evaluate the severity of sarcopenia and guide clinical practice to some extent. Our previous multicenter research revealed that the sarcopenia was a predictive factor for postoperative complications in HAE patients undergoing liver resection and 
Points

$\begin{array}{llllllllll}0 & 10 & 20 & 30 & 40 & 50 & 60 & 70 & 80 & 90\end{array}$

Duration From Primary

Diagnosis To Obvious

Symptoms

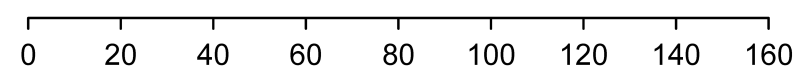

Surgical Time

$400 \quad 600 \quad 800 \quad 1000 \quad 1200$

Sarcopenia Score

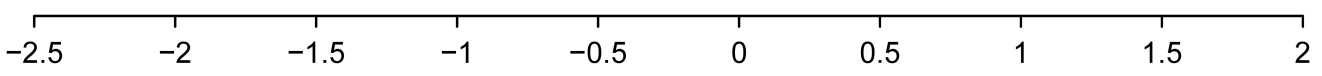

Total Points

\begin{tabular}{lllllllllll}
\hline & 20 & 40 & 60 & 80 & 100 & 120 & 140 & 160 & 180
\end{tabular}

Linear Predictor

\begin{tabular}{ll|ll}
\hline-2 & 1 & 4 & 6
\end{tabular}

Major Postoperative Complication

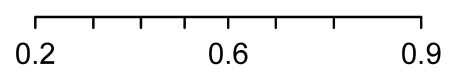

Figure 2 Nomogram for predicting major postoperative complications (Clavien-Dindo $\geq$ IIla). The nomogram represents the predicted probability of major postoperative complications on a scale of 0 to 180 . For each predictor, draw a vertical line straight up to the point axis and note the corresponding points. Sum the points from each predictor, and the total score corresponding to a predicted probability of major postoperative complications can be found at the bottom of the nomogram.

applied the severity of sarcopenia as a classifier to divide all patients into two groups for prediction. ${ }^{28}$ In contrast, we retained the original sarcopenia score model to maintain a higher AUROC for the final nomogram for more accurate prediction.

Studies have reported that sarcopenia might be induced by complicated genetic and environmental factors. A study on an aged population showed that $\mathrm{C}$-reactive protein (CRP) and interleukin-6 (IL-6) increase the risk for loss of muscle mass and muscle strength due to the imbalance of muscle synthesis and increased protein catabolism. ${ }^{63}$ Recently, a study on twins showed that variations in muscle quantity was associated with the methylation of genes. ${ }^{64}$ It is generally believed that the poor basic physical state resulting from sarcopenia may reduce immune function and tolerance to large trauma or surgeries such as ELRA. ${ }^{65}$ Adipose tissue, which has been indicated to be related to chronic inflammation of the body and increased proinflammatory cytokines, ${ }^{66}$ was also included when evaluating the sarcopenia score in our study. It has been shown in a study about gastric carcinoma that visceral fat area is associated with multiple postoperative complications, especially abdominal abscess and pancreatic fistula. ${ }^{67}$ Some researchers regard visceral adipose tissue 
A

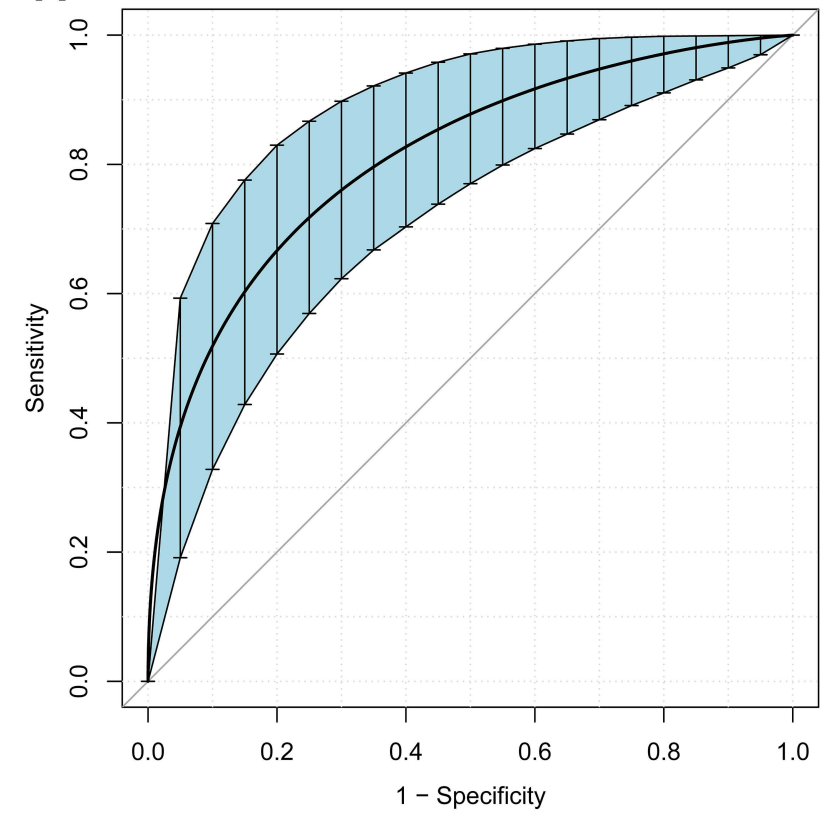

B

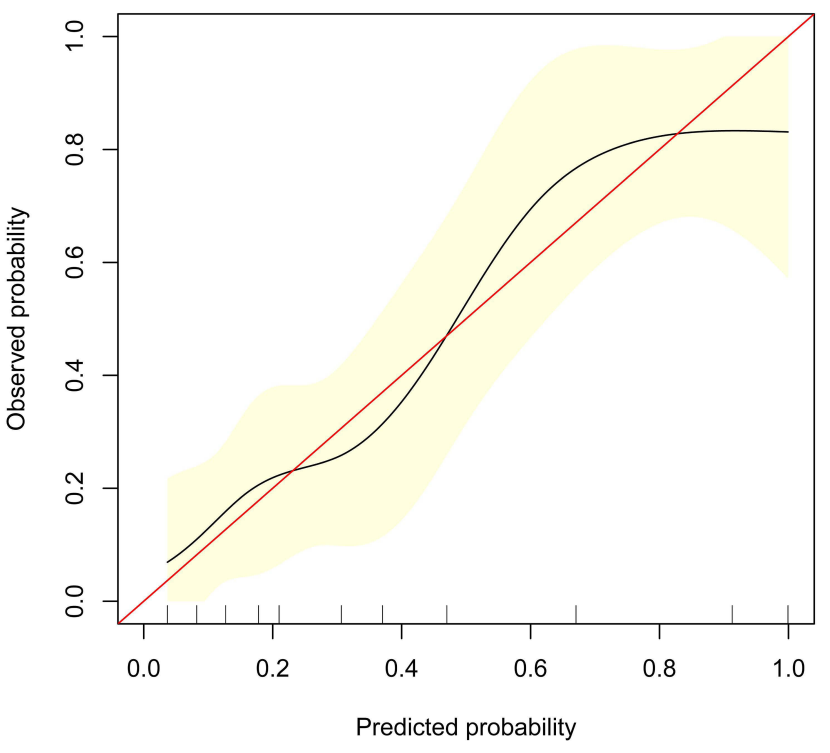

Figure 3 Receiver operating characteristic (ROC) curve and calibration curve for predicting major postoperative complications (Clavien-Dindo grade $\geq$ Illa). (A) ROC curve for the prediction model obtained by the bootstrapping method (resample: 500 ), with an area under the receiver operating characteristic curve (AUROC) of 0.807 ( $95 \% \mathrm{Cl}$, 0.720-0.895). (B) Calibration curve for the prediction model. The X-axis represents the predicted probability of major postoperative complications according to the prediction model. The vertical black lines on the $\mathrm{X}$-axis represent the distribution of the samples. The Y-axis represents the observed probability of major postoperative complications. The red line shows an ideal prediction corresponding to the actual observations. The black line represents the performance of the calibration curve with the bootstrapping method (resample: 500).

as an endocrine organ that can secrete IL-6, tumor necrosis factor- $\alpha$ (TNF- $\alpha)$, and others to further cause a proinflammatory status. ${ }^{68}$

As expected, the surgical time of ELRA was selected as an independent risk factor for major postoperative complications. In 2020, Giustiniano et $\mathrm{al}^{69}$ and Cezar et al ${ }^{70}$ found that surgical time was associated with postoperative complications of liver resection and could predict acute kidney injury after liver transplantation, which is similar to our results. Therefore, we should strictly comply with aseptic principles and further improve our surgical efficiency to minimize postoperative complications. Interestingly, the duration from primary diagnosis to obvious symptoms was also selected as a predictive factor for postoperative complications by multivariate logistic regression. As previously described, HAE has the characteristic of insidious onset and tends to reach an end stage before being diagnosed. Undoubtfully, invasion into crucial intrahepatic structures and local metastasis caused by disease progression often increase the difficulty of the operation and even affect patient prognosis. Hence, it is particularly important to focus on the early diagnosis and early treatment of HAE while exploring surgical methods.
To the best of our knowledge, this is the first study thus far that reports the relationship between sarcopenia and the postoperative complications of ELRA. Furthermore, our findings have important clinical implications. Specifically, the calculator (Supplementary file 1) can provide clinicians and patients with sarcopenia score as well as predicted risk of major postoperative complications after ELRA for end-stage HAE patients, which can help and guide clinical decision making in this population. For high-risk end-stage HAE patients, preoperative nutritional status and physical fitness should be improved. In addition, technical efficiency and proficiency in performing ELRA are of crucial importance in the improvement of prognosis. Early diagnosis and early treatment of HAE should also be particularly valued.

However, this study should be interpreted in light of some inherent limitations. First, as a linear regression shrinkage method, LASSO regression shrinks the regression coefficient within a certain area and constructs a first-order punishment function to obtain a refined model. Whereas LASSO regression model does not fully reflect the significance of the included variables. The LASSO model of sarcopenia score only demonstrated that postoperative 
Table 3 Clinical Data and Surgical Characteristics of the Patients in the Low-Risk Group and the High-Risk Group

\begin{tabular}{|c|c|c|c|}
\hline Variable & Low-Risk Group & High-Risk Group & $\mathbf{P}$ \\
\hline \multicolumn{4}{|c|}{ Baseline data } \\
\hline Number of patients, $\mathrm{n}(\%)$ & $52(51.49 \%)$ & $49(48.51 \%)$ & \\
\hline Age (years), mean \pm SD & $33.27 \pm 9.94$ & $40.96 \pm 8.42$ & $<0.001$ \\
\hline BMI $\left(\mathrm{kg} / \mathrm{m}^{2}\right)$, mean $\pm \mathrm{SD}$ & $21.98 \pm 2.98$ & $22.13 \pm 3.46$ & 0.813 \\
\hline Duration from primary diagnosis to obvious symptoms (months), mean $\pm S D$ & $15.65 \pm 13.12$ & $46.88 \pm 39.76$ & $<0.001$ \\
\hline Male sex, n (\%) & $22(42.31 \%)$ & $16(32.65 \%)$ & 0.317 \\
\hline Previous use of oral albendazole, $n(\%)$ & $31(59.62 \%)$ & $34(69.39 \%)$ & 0.305 \\
\hline History of jaundice, $\mathrm{n}(\%)$ & $8(15.39 \%)$ & $10(20.41 \%)$ & 0.510 \\
\hline \multicolumn{4}{|c|}{ Preoperative data of skeletal muscle and adipose tissue } \\
\hline $\mathrm{PMI}\left(\mathrm{cm}^{2} / \mathrm{m}^{2}\right)$, mean $\pm \mathrm{SD}$ & $6.25 \pm|.7|$ & $4.82 \pm 2.17$ & $<0.001$ \\
\hline $\mathrm{SMI}\left(\mathrm{cm}^{2} / \mathrm{m}^{2}\right)$, mean $\pm \mathrm{SD}$ & $44.15 \pm 6.69$ & $38.38 \pm 9.18$ & $<0.001$ \\
\hline VSR $\left(\mathrm{cm}^{2} / \mathrm{cm}^{2}\right)$, mean \pm SD & $0.21 \pm 0.10$ & $0.35 \pm 0.21$ & $<0.001$ \\
\hline IMAC (HU/HU), mean \pm SD & $-0.64 \pm 0.17$ & $-0.49 \pm 0.16$ & $<0.001$ \\
\hline Sarcopenia score, mean \pm SD & $-0.88 \pm 0.45$ & $-0.09 \pm 0.60$ & $<0.001$ \\
\hline \multicolumn{4}{|c|}{ Preoperative data of the lesions } \\
\hline Lesion diameter $(\mathrm{cm})$, mean $\pm \mathrm{SD}$ & $14.77 \pm 2.87$ & $\mid 5.59 \pm 2.51$ & 0.129 \\
\hline Surrounding invasion, n (\%) & $30(57.69 \%)$ & $38(77.55 \%)$ & 0.033 \\
\hline Distant metastasis, n (\%) & $5(9.62 \%)$ & II (22.45\%) & 0.077 \\
\hline Number of lesions, $n$ (\%) & & & 0.512 \\
\hline Single & $32(61.54 \%)$ & $27(55.10 \%)$ & \\
\hline Multiple & $20(38.46 \%)$ & $22(44.90 \%)$ & \\
\hline Inferior vena cava invasion, $\mathrm{n}(\%)$ & & & 0.714 \\
\hline I & 5 (9.62\%) & $3(6.12 \%)$ & \\
\hline II & $38(73.08 \%)$ & 39 (79.59\%) & \\
\hline III & $9(17.31 \%)$ & 7 (14.29\%) & \\
\hline Hepatic vein invasion, $\mathrm{n}(\%)$ & & & 0.454 \\
\hline I & $24(46.15 \%)$ & $19(38.78 \%)$ & \\
\hline II & $28(53.85 \%)$ & $30(61.22 \%)$ & \\
\hline Portal vein invasion, $\mathrm{n}(\%)$ & & & 0.049 \\
\hline I & $4(7.69 \%)$ & $3(6.12 \%)$ & \\
\hline II & 40 (76.92\%) & $28(57.14 \%)$ & \\
\hline III & $8(15.38 \%)$ & $18(36.73 \%)$ & \\
\hline \multicolumn{4}{|c|}{ Preoperative data of liver volume and quality } \\
\hline $\operatorname{ERLV}(\mathrm{mL})$, mean $\pm \mathrm{SD}$ & $680.67 \pm 187.16$ & $747.00 \pm 206.61$ & 0.094 \\
\hline $\mathrm{SLV}(\mathrm{mL})$, mean $\pm \mathrm{SD}$ & $1140.15 \pm 107.89$ & $1155.55 \pm 111.07$ & 0.482 \\
\hline ERLV/SLV, mean \pm SD & $0.60 \pm 0.16$ & $0.65 \pm 0.19$ & 0.125 \\
\hline Child-Pugh classification, n (\%) & & & 0.443 \\
\hline Class A & $48(92.31 \%)$ & 47 (95.92\%) & \\
\hline Class B & $4(7.69 \%)$ & $2(4.08 \%)$ & \\
\hline Albumin-bilirubin grade, $n$ (\%) & & & 0.129 \\
\hline Grade I $(\leq-2.60)$ & $15(28.85 \%)$ & $8(16.33 \%)$ & \\
\hline Grade $2(>-2.60, \leq-1.39)$ & 37 (7I.15\%) & 39 (79.59\%) & \\
\hline Grade $3(>-1.39)$ & $0(0.00 \%)$ & $2(4.08 \%)$ & \\
\hline
\end{tabular}


Table 3 (Continued).

\begin{tabular}{|c|c|c|c|}
\hline Variable & Low-Risk Group & High-Risk Group & $\mathbf{P}$ \\
\hline \multicolumn{4}{|c|}{ Intraoperative data } \\
\hline $\operatorname{ARLV}(m L)$, mean $\pm S D$ & $686.02 \pm 185.46$ & $762.27 \pm 224.37$ & 0.065 \\
\hline ARLV/SLV, mean \pm SD & $0.60 \pm 0.15$ & $0.66 \pm 0.19$ & 0.079 \\
\hline Surgical time $(\mathrm{min})$, mean $\pm \mathrm{SD}$ & $677.52 \pm 89.73$ & $816.08 \pm 143.23$ & $<0.001$ \\
\hline Anhepatic time (30 min), mean $\pm S D$ & $9.20 \pm 2.45$ & $11.54 \pm 2.24$ & $<0.001$ \\
\hline Hemodynamic instability time $(\mathrm{min})$, mean \pm SD & $140.87 \pm 28.82$ & $157.37 \pm 39.63$ & 0.018 \\
\hline Liver graft reimplantation time ( $10 \mathrm{~min})$, mean \pm SD & $5.63 \pm 2.34$ & $6.30 \pm 2.48$ & 0.169 \\
\hline Blood loss $(\mathrm{mL})$, mean $\pm \mathrm{SD}$ & $2416.35 \pm 1078.48$ & $3088.78 \pm 2245.63$ & 0.056 \\
\hline Autologous blood transfusion ( $100 \mathrm{~mL})$, mean $\pm \mathrm{SD}$ & $8.77 \pm 7.42$ & $9.96 \pm 11.83$ & 0.546 \\
\hline RBC suspension transfusion $(U)$, mean $\pm S D$ & $5.50 \pm 4.10$ & $42.49 \pm 227.29$ & 0.243 \\
\hline Fresh frozen plasma transfusion $(\mathrm{mL})$, mean $\pm \mathrm{SD}$ & $654.23 \pm 650.28$ & $1224.69 \pm 1232.82$ & 0.004 \\
\hline Combined resection, $\mathrm{n}(\%)$ & $8(15.38 \%)$ & 14 (28.57\%) & 0.109 \\
\hline \multicolumn{4}{|c|}{ Length of stay and postoperative complications } \\
\hline Length of stay in the hospital (days), median (range) & $20.00(15.00-30.25)$ & $22.00(15.00-33.00)$ & 0.671 \\
\hline Length of stay in the ICU (days), median (range) & $4.00(3.00-5.00)$ & $4.00(3.00-5.00)$ & 0.014 \\
\hline Postoperative complication, n (\%) & $21(40.38 \%)$ & $36(73.47 \%)$ & $<0.001$ \\
\hline Postoperative complication (Clavien-Dindo $\geq \mathrm{IIla}$ ), n (\%) & $8(15.38 \%)$ & $31(63.27 \%)$ & $<0.001$ \\
\hline $\mathrm{CCl}$, median (range) & $0.00(0.00-20.92)$ & $26.22(0.00-33.73)$ & $<0.001$ \\
\hline
\end{tabular}

Abbreviations: BMI, body mass index; PMI, psoas muscle mass index; SMI, skeletal muscle mass index; VSR, visceral to subcutaneous adipose tissue area ratio; IMAC, intramuscular adipose tissue content; ERLV, estimated remnant liver volume; SLV, standard liver volume; ARLV, actual remnant liver volume; RBC, red blood cell; ICU, intensive care unit; $\mathrm{CCl}$, comprehensive complication index.

complications of ELRA were associated with SMI, VSR, IMAC and age in this study. Therefore, readers should interpret the results of LASSO regression with caution. Second, our conclusions are based on a special and relatively rare parasitic disease, and the number of cases is relatively small when compared with other hepatic diseases. Therefore, the selection of statistical methods and extrapolation of our results are subject to limitations, and selection bias is inevitable Third, this is a retrospective study that was conducted in a single center. Despite the limited number of patients and the high degree of difficulty of ELRA, further prospective and multicenter studies are required.

In conclusion, we analyzed the largest number of ELRA patients to date and provided a method to evaluate sarcopenia score that was an integrated preoperative assessment of skeletal muscle and adipose tissue in endstage HAE patients. A nomogram incorporating the duration from primary diagnosis to obvious symptoms, surgical time and sarcopenia score was then constructed to predict major postoperative complications of ELRA and thus provide hints for preoperative nutritional intervention and complication prevention.

\section{Data Sharing Statement}

The datasets generated and analyzed during the current study are available from the corresponding author on reasonable request.

\section{Ethics Approval and Informed Consent}

The study was conducted in accordance with the Declaration of Helsinki and was approved by the Ethics Committee of West China Hospital of Sichuan University (No. 2017-38). The requirement for informed consent was waived because the study was retrospective, and the data were anonymized.

\section{Author Contributions}

All authors made a significant contribution to the work reported, whether that is in the conception, study design, execution, acquisition of data, analysis and interpretation, or in all these areas; have drafted, revised or critically reviewed the article; read and approved the final manuscript; have agreed on the journal to which the article 
would be submitted; and agree to take responsibility for the contents of the article.

\section{Funding}

This research was supported by the Science and Technology Program of Sichuan Science and Technology Department (No. 2019YFS0029, 2019YFS0529), the National Natural Science Foundation of China (No. 81770566, 82170543, 82000599) and the New Medical Technology Foundation of West China Hospital of Sichuan University (No. XJS2016004). Corresponding author Wentao Wang and Shu Shen are the guarantors. The funding body financed the costs of the study and contributed to the design of the study, interpretation of data, and revising the manuscript.

\section{Disclosure}

The authors report no conflict of interest.

\section{References}

1. Shang JY, Zhang GJ, Liao S, et al. A multiplex PCR for differential detection of Echinococcus granulosus sensu stricto, Echinococcus multilocularis and Echinococcus canadensis in China. Infect Dis Poverty. 2019;8(1):68. doi:10.1186/s40249-019-0580-2

2. Wen H, Vuitton L, Tuxun T, et al. Echinococcosis: advances in the 21st century. Clin Microbiol Rev. 2019;32(2). doi:10.1128/ CMR.00075-18

3. Siles-Lucas M, Casulli A, Conraths FJ, Muller N. Laboratory diagnosis of echinococcus spp. in human patients and infected animals. Adv Parasitol. 2017;96:159-257. doi:10.1016/bs.apar.2016.09.003

4. Vuitton D. Alveolar echinococcosis of the liver: a parasitic disease in search of a treatment. Hepatology. 1990;12(3 Pt 1):617-618. doi:10.1002/hep. 1840120329

5. Craig P. Echinococcus multilocularis. Curr Opin Infect Dis. 2003;16 (5):437-444. doi:10.1097/00001432-200310000-00010

6. Qiu Y, Yang X, Shen S, Huang B, Wang W. Vascular infiltration-based surgical planning in treating end-stage hepatic alveolar echinococcosis with ex vivo liver resection and autotransplantation. Surgery. 2019;165(5):889-896. doi:10.1016/j. surg.2018.11.007

7. Yang X, Qiu Y, Huang B, et al. Novel techniques and preliminary results of ex vivo liver resection and autotransplantation for end-stage hepatic alveolar echinococcosis: a study of 31 cases. $\mathrm{Am}$ J Transplant. 2018;18(7):1668-1679. doi:10.1111/ajt.14621

8. Shen S, Qiu Y, Yang X, Wang W. Remnant liver-to-standard liver volume ratio below $40 \%$ is safe in ex vivo liver resection and autotransplantation. J Gastrointest Surg. 2019;23(10):1964-1972. doi:10.1007/s11605-018-4022-4

9. Pichlmayr R, Grosse H, Hauss J, Gubernatis G, Lamesch P, Bretschneider HJ. Technique and preliminary results of extracorporeal liver surgery (bench procedure) and of surgery on the in situ perfused liver. $B r \quad J$ Surg. 1990;77(1):21-26. doi:10.1002/ bjs. 1800770107

10. Zawistowski M, Nowaczyk J, Jakubczyk M, Domagala P. Outcomes of ex vivo liver resection and autotransplantation: a systematic review and meta-analysis. Surgery. 2020;168(4):631-642. doi:10.1016/j.surg.2020.05.036
11. Aji T, Dong JH, Shao YM, et al. Ex vivo liver resection and autotransplantation as alternative to allotransplantation for end-stage hepatic alveolar echinococcosis. J Hepatol. 2018;69(5):1037-1046. doi:10.1016/j.jhep.2018.07.006

12. Probst P, Fuchs J, Schon MR, et al. Prospective study to evaluate the prognostic value of different nutritional assessment scores in liver surgery: NURIMAS Liver (DRKS00006340). Hepatobiliary Surg Nutr. 2020;9(4):400-413. doi:10.21037/hbsn.2019.06.11

13. Lobo DN, Gianotti L, Adiamah A, et al. Perioperative nutrition: recommendations from the ESPEN expert group. Clin Nutr. 2020;39(11):3211-3227. doi:10.1016/j.clnu.2020.03.038

14. Cruz-Jentoft AJ, Bahat G, Bauer J, et al. Sarcopenia: revised European consensus on definition and diagnosis. Age Ageing. 2019;48(1):16-31. doi:10.1093/ageing/afy169

15. Kalafateli M, Mantzoukis K, Choi YY, et al. Malnutrition and sarcopenia predict post-liver transplantation outcomes independently of the model for end-stage liver disease score. J Cachexia Sarcopenia Muscle. 2017;8(1):113-121. doi:10.1002/jcsm.12095

16. Bhanji RA, Takahashi N, Moynagh MR, et al. The evolution and impact of sarcopenia pre- and post-liver transplantation. Aliment Pharmacol Ther. 2019;49(6):807-813. doi:10.1111/apt.15161

17. Nam NH, Kaido T, Uemoto S. Assessment and significance of sarcopenia in liver transplantation. Clin Transplant. 2019;33(12). doi:10.1111/ctr.13741

18. Hamaguchi Y, Kaido T, Okumura S, et al. Impact of skeletal muscle mass index, intramuscular adipose tissue content, and visceral to subcutaneous adipose tissue area ratio on early mortality of living donor liver transplantation. Transplantation. 2017;101(3):565-574. doi:10.1097/TP.0000000000001587

19. Fujiwara N, Nakagawa H, Kudo Y, et al. Sarcopenia, intramuscular fat deposition, and visceral adiposity independently predict the outcomes of hepatocellular carcinoma. J Hepatol. 2015;63(1):131-140. doi:10.1016/j.jhep.2015.02.031

20. Okumura S, Kaido T, Hamaguchi Y, et al. Impact of skeletal muscle mass, muscle quality, and visceral adiposity on outcomes following resection of intrahepatic cholangiocarcinoma. Ann Surg Oncol. 2017;24(4):1037-1045. doi:10.1245/s10434-016-5668-3

21. Englesbe MJ, Patel SP, He K, et al. Sarcopenia and mortality after liver transplantation. $J$ Am Coll Surg. 2010;211(2):271-278. doi:10.1016/j.jamcollsurg.2010.03.039

22. Nakamura $R$, Inage $Y$, Tobita $R$, et al. Sarcopenia in resected NSCLC: effect on postoperative outcomes. J Thorac Oncol. 2018;13(7):895-903. doi:10.1016/j.jtho.2018.04.035

23. Joglekar S, Asghar A, Mott SL, et al. Sarcopenia is an independent predictor of complications following pancreatectomy for adenocarcinoma. J Surg Oncol. 2015;111(6):771-775. doi:10.1002/ jso. 23862

24. Simonsen C, de Heer P, Bjerre ED, et al. Sarcopenia and postoperative complication risk in gastrointestinal surgical oncology: a meta-analysis. Ann Surg. 2018;268(1):58-69. doi:10.1097/ SLA.0000000000002679

25. Hsu CS, Kao JH. Sarcopenia and chronic liver diseases. Expert Rev Gastroenterol Hepatol. 2018;12(12):1229-1244. doi:10.1080/ 17474124.2018.1534586

26. Ooi PH, Hager A, Mazurak VC, et al. Sarcopenia in chronic liver disease: impact on outcomes. Liver Transpl. 2019;25(9):1422-1438. doi:10.1002/lt.25591

27. Czigany Z, Kramp W, Bednarsch J, et al. Myosteatosis to predict inferior perioperative outcome in patients undergoing orthotopic liver transplantation. Am J Transplant. 2020;20(2):493-503. doi:10.1111/ ajt.15577

28. Wang T, Yang X, Wang W, et al. A new sarcopenia score prognostic for postoperative complications in hepatic alveolar echinococcosis: a multicenter retrospective study. Ann Transl Med. 2020;8(21):1398. doi:10.21037/atm-20-1960a 
29. Dindo D, Demartines N, Clavien PA. Classification of surgical complications: a new proposal with evaluation in a cohort of 6336 patients and results of a survey. Ann Surg. 2004;240(2):205-213.

30. Yamashita Y, Hamatsu T, Rikimaru T, et al. Bile leakage after hepatic resection. Ann Surg. 2001;233(1):45-50. doi:10.1097/00000658200101000-00008

31. Ishii M, Mizuguchi T, Harada K, et al. Comprehensive review of post-liver resection surgical complications and a new universal classification and grading system. World J Hepatol. 2014;6(10):745-751. doi:10.4254/wjh.v6.i10.745

32. Tsai KY, Chen HA, Wang WY, Huang MT. Risk factors analysis of postoperative pleural effusion after liver resection. Dig Surg. 2019;36 (6):514-521. doi:10.1159/000494218

33. Kern P, Wen H, Sato N, et al. WHO classification of alveolar echinococcosis: principles and application. Parasitol Int. 2006;55 Suppl:S283-7. doi:10.1016/j.parint.2005.11.041

34. He YB, Bai L, Jiang Y, et al. Application of a three-dimensional reconstruction technique in liver autotransplantation for end-stage hepatic alveolar echinococcosis. J Gastrointest Surg. 2015;19 (8):1457-1465. doi:10.1007/s11605-015-2842-Z

35. Tongyoo A, Pomfret EA, Pomposelli JJ. Accurate estimation of living donor right hemi-liver volume from portal vein diameter measurement and standard liver volume calculation. Am J Transplant. 2012;12(5):1229-1239. doi:10.1111/j.1600-6143.2011.03909.x

36. Urata K, Kawasaki S, Matsunami H, et al. Calculation of child and adult standard liver volume for liver transplantation. Hepatology. 1995;21(5):1317-1321. doi:10.1002/hep.1840210515

37. Mourtzakis M, Prado CM, Lieffers JR, Reiman T, McCargar LJ, Baracos VE. A practical and precise approach to quantification of body composition in cancer patients using computed tomography images acquired during routine care. Appl Physiol Nutr Metab. 2008;33(5):997-1006. doi:10.1139/H08-075

38. Clavien PA, Barkun J, de Oliveira ML, et al. The Clavien-Dindo classification of surgical complications: five-year experience. Ann Surg. 2009;250(2):187-196. doi:10.1097/SLA.0b013e3181b13ca2

39. Slankamenac K, Graf R, Barkun J, Puhan MA, Clavien PA. The comprehensive complication index: a novel continuous scale to measure surgical morbidity. Ann Surg. 2013;258(1):1-7. doi:10.1097/ SLA.0b013e318296c732

40. Torgerson PR, Keller K, Magnotta M, Ragland N. The global burden of alveolar echinococcosis. PLoS Negl Trop Dis. 2010;4(6):e722. doi:10.1371/journal.pntd.0000722

41. Bresson-Hadni S, Vuitton DA, Bartholomot B, et al. A twenty-year history of alveolar echinococcosis: analysis of a series of 117 patients from eastern France. Eur $J$ Gastroenterol Hepatol. 2000;12 (3):327-336. doi:10.1097/00042737-200012030-00011

42. Kern P. Clinical features and treatment of alveolar echinococcosis. Curr Opin Infect Dis. 2010;23(5):187-196. doi:10.1097/ QCO.0b013e32833d7516

43. Qu B, Guo L, Sheng G, et al. Management of advanced hepatic alveolar echinococcosis: report of 42 cases. Am J Trop Med Hyg. 2017;96(3):680-685. doi:10.4269/ajtmh.16-0557

44. Ozdemir F, Ince V, Barut B, Onur A, Kayaalp C, Yilmaz S. Living donor liver transplantation for Echinococcus Alveolaris: single-center experience. Liver Transpl. 2015;21(8):1091-1095. doi:10.1002/1t.24170

45. Bresson-Hadni S, Blagosklonov O, Knapp J, et al. Should possible recurrence of disease contraindicate liver transplantation in patients with end-stage alveolar echinococcosis? A 20 -year follow-up study. Liver Transpl. 2011;17(7):855-865. doi:10.1002/1t.22299

46. Chapuis Y, Houssin D, Brouzes S, Ortega D. [Hepatic transplantation in alveolar echinococcosis. 3 attempts]. Chirurgie. 1987;113 (6):634-640. French.
47. Bresson-Hadni S, Koch S, Miguet JP, et al. Indications and results of liver transplantation for Echinococcus alveolar infection: an overview. Langenbecks Arch Surg. 2003;388(4):231-238. doi:10.1007/s00423-003-0394-2

48. Wen H, Dong JH, Zhang JH, et al. Ex vivo liver resection followed by autotransplantation for end-stage hepatic alveolar echinococcosis. Chin Med J. 2011;124(18):2813-2817.

49. Zhang Y, Lai E, Yang C, et al. In situ reconstruction of vascular inflow/outflow to left lateral liver section, ex-vivo liver resection and autologous liver transplantation of remaining liver remnant for hepatic alveolar echinococcosis. Int J Surg Case Rep. 2020;69:39-43. doi:10.1016/j.ijscr.2020.03.023

50. Aini A, Shao Y, Shalayiadang P, et al. Auxiliary partial autologous liver transplantation for high-selective alveolar echinococcosis: a proof of concept. Transplantation. 2020;104(5):e138-9. doi:10.1097/TP.0000000000003092

51. Jianyong L, Jingcheng H, Wentao W, et al. Ex vivo liver resection followed by autotransplantation to a patient with advanced alveolar echinococcosis with a replacement of the retrohepatic inferior vena cava using autogenous vein grafting: a case report and literature review. Medicine. 2015;94(7):e514. doi:10.1097/ MD.0000000000000514

52. Wang H, Liu Q, Wang Z, Zhang F, Li X, Wang X. Clinical outcomes of Ex Vivo liver resection and liver autotransplantation for hepatic alveolar echinococcosis. J Huazhong Univ Sci Technolog Med Sci. 2012;32(4):598-600. doi:10.1007/s11596-012-1003-9

53. Khuri SF, Henderson WG, DePalma RG, Mosca C, Healey NA, Kumbhani DJ. Determinants of long-term survival after major surgery and the adverse effect of postoperative complications. Ann Surg. 2005;242(3):326-41, 341-3. doi:10.1097/01. sla.0000179621.33268.83

54. Cruz-Jentoft AJ, Baeyens JP, Bauer JM, et al. Sarcopenia: European consensus on definition and diagnosis: report of the European working group on sarcopenia in older people. Age Ageing. 2010;39 (4):412-423. doi:10.1093/ageing/afq034

55. Kobayashi A, Kaido T, Hamaguchi Y, et al. Impact of sarcopenic obesity on outcomes in patients undergoing hepatectomy for hepatocellular carcinoma. Ann Surg. 2019;269(5):924-931. doi:10.1097/ SLA.0000000000002555

56. Harimoto N, Hoshino H, Muranushi R, et al. Skeletal muscle volume and intramuscular adipose tissue are prognostic predictors of postoperative complications after hepatic resection. Anticancer Res. 2018;38(8):4933-4939. doi:10.21873/anticanres. 12810

57. Lou N, Chi CH, Chen XD, et al. Sarcopenia in overweight and obese patients is a predictive factor for postoperative complication in gastric cancer: a prospective study. Eur J Surg Oncol. 2017;43 (1):188-195. doi:10.1016/j.ejso.2016.09.006

58. Hamaguchi Y, Kaido T, Okumura S, et al. Muscle steatosis is an independent predictor of postoperative complications in patients with hepatocellular carcinoma. World J Surg. 2016;40(8):1959-1968. doi:10.1007/s00268-016-3504-3

59. Golse N, Bucur PO, Ciacio O, et al. A new definition of sarcopenia in patients with cirrhosis undergoing liver transplantation. Liver Transpl. 2017;23(2):143-154. doi:10.1002/1t.24671

60. Hamaguchi Y, Kaido T, Okumura S, et al. Proposal of muscle-MELD score, including muscularity, for prediction of mortality after living donor liver transplantation. Transplantation. 2016;100 (11):2416-2423. doi:10.1097/TP.0000000000001413

61. Meza-Junco J, Montano-Loza AJ, Baracos VE, et al. Sarcopenia as a prognostic index of nutritional status in concurrent cirrhosis and hepatocellular carcinoma. J Clin Gastroenterol. 2013;47 (10):861-870. doi:10.1097/MCG.0b013e318293a825 
62. Hamaguchi Y, Kaido T, Okumura S, et al. Impact of quality as well as quantity of skeletal muscle on outcomes after liver transplantation. Liver Transpl. 2014;20(11):1413-1419. doi:10.1002/lt.23970

63. Visser M, Pahor M, Taaffe DR, et al. Relationship of interleukin-6 and tumor necrosis factor-alpha with muscle mass and muscle strength in elderly men and women: the Health ABC Study. J Gerontol a Biol Sci Med Sci. 2002;57(5):M326-32. doi:10.1093/ gerona/57.5.M326

64. Livshits G, Gao F, Malkin I, et al. Contribution of heritability and epigenetic factors to skeletal muscle mass variation in United Kingdom twins. J Clin Endocrinol Metab. 2016;101(6):2450-2459. doi: $10.1210 /$ jc. $2016-1219$

65. Prado CM, Lieffers JR, McCargar LJ, et al. Prevalence and clinical implications of sarcopenic obesity in patients with solid tumours of the respiratory and gastrointestinal tracts: a population-based study. Lancet Oncol. 2008;9(7):629-635. doi:10.1016/S1470-2045(08) 70153-0
66. Dalamaga M. Interplay of adipokines and myokines in cancer pathophysiology: emerging therapeutic implications. World J Exp Med. 2013;3(3):26-33. doi:10.5493/wjem.v3.i3.26

67. Okada K, Nishigori T, Obama K, et al. The incidence of postoperative complications after gastrectomy increases in proportion to the amount of preoperative visceral fat. J Oncol. 2019;2019:8404383. doi:10.1155/2019/8404383

68. Fernandes JV, Cobucci RN, Jatoba CA, Fernandes TA, de Azevedo JW, de Araujo JM. The role of the mediators of inflammation in cancer development. Pathol Oncol Res. 2015;21(3):527-534. doi:10.1007/s12253-015-9913-z

69. Giustiniano E, Procopio F, Morenghi E, et al. Renal resistive index as a predictor of postoperative complications in liver resection surgery. Observational study. J Clin Monit Comput. 2020;35:731-740.

70. Cezar LC, Meneses GC, Da SJG, et al. Intraoperative systemic biomarkers predict post-liver transplantation acute kidney injury. Eur J Gastroenterol Hepatol. 2020;33:1556-1563.

\section{Publish your work in this journal}

Infection and Drug Resistance is an international, peer-reviewed openaccess journal that focuses on the optimal treatment of infection (bacterial, fungal and viral) and the development and institution of preventive strategies to minimize the development and spread of resistance. The journal is specifically concerned with the epidemiology of antibiotic resistance and the mechanisms of resistance development and diffusion in both hospitals and the community. The manuscript management system is completely online and includes a very quick and fair peerreview system, which is all easy to use. Visit http://www.dovepress.com/ testimonials.php to read real quotes from published authors. 